\title{
Stygocladius multisetosus gen. nov., sp. nov., a rheophilic element, inhabiting basaltic and karstic helocrenes in France and Algeria (Diptera: (hironomidae)
}

\author{
Joel Moubayed-Breil', Patrick Ashe ${ }^{2}$ and Peter H. Langton ${ }^{3}$
}

\begin{abstract}
Moubayed-Breil J, Ashe P and Langton PH. 2012. Stygocladius multisetosus gen. nov., sp. nov., a rheophilic element, inhabiting basaltic and karstic helocrenes in France and Algeria (Diptera: Chironomidae). Fauna norvegica 31: 175-182.
\end{abstract}

\begin{abstract}
Diagnoses and descriptions of the adults and pupal exuviae of Stygocladius multisetosus gen. nov., sp. nov., are provided based primarily on male and female pharates collected in temporary basaltic and karstic helocrenes in southern France and Algeria. Features on the adult male, female and pupa indicates that it belongs to the Heterotrissocladius group of genera and is probably most closely related to Paratrissocladius Zavřel. The immature stages of the new species are stygobiontic.
\end{abstract}

doi: 10.5324/fn.v31i0.1363. Received: 2011-10-09. Accepted: 2012-06-29.

Published on paper and online: 2012-10-17.

Keywords: Chironomidae, Orthocladiinae, Stygocladius, new genus, new species, helocrenes, Algeria, France

1. Applied ecology, 10 rue des Fenouils, F-34070 Montpellier, France

2. 33 Shelton Drive, Terenure, Dublin 12, Ireland

3. 16 Irish Society Court, Coleraine, Co. Londonderry, BT52 1GX Northern Ireland

Corresponding author: Joel Moubayed-Breil

E-mail:jm.aquabiol@neuf.fr

\section{INTRODUCTION}

Pupal exuviae of a strange orthoclad, with an anal lobe bearing both a fringe and more than three anal macrosetae, were first found by the senior author in the south of France in 1994. The rare combination of these two features was then only known to occur in the exuviae of Psectrocladius (Psectrocladius) barbimanus (Edwards, 1929) and in species of the genus Propsilocerus Kieffer, 1923. One further species, Hydrobaenus olfa Zerguine \& Rossaro, 2010 has since been described with the same exuvial features (Zerguine \& Rossaro 2010). However, other characters on the exuviae of the French specimens, subsequently discovered in Algeria in 2001, do not match the descriptions of any of those taxa listed above. The new species described here has been previously reported as Heterotrissocladius sp. 1 from southern France (Moubayed 1998; Moubayed-Breil 2007) and Algeria (Moubayed-Breil et al. 2007). In this paper a new genus is created, based on diagnoses of the adult and pupal stages, to accommodate the new species. Occurrence of the new species in southern France and Algeria indicates that it is likely to be more widespread and can at least be expected from helocrenes habitats in the Tyrrhenian subregion which includes: eastern Spain, western Italy, Tunisia, Morocco and some of the larger western Mediterranean islands. Survival and growth of the immature stages within helocrenes are influenced by an annual rhythm between a short wet season and a long dry season.

\section{MATERIAL AND METHODS}

The adult specimens were cleared in $90 \%$ lactic acid which took a minimum of 20-30 minutes - material can be left overnight at room temperature without any detrimental effect or damage. When clearing was complete the specimens were washed in 
two changes of $70 \%$ ethanol to ensure that all traces of lactic acid were removed. Compared to clearing with potassium hydroxide, or other clearing solutions, no deterioration of the typical "original" structure is reported by using lactic acid. More details on the above technique will be given in MoubayedBreil \& Ashe (in prep.). All examined material (adults and pupal exuviae) was mounted in polyvinyl lactophenol. Before the final slide preparation was made the hypopygium was viewed laterally (to draw a side view of the anal point) and ventrally to examine or draw the virga before the hypopygium was turned into its permanent dorsal position and covered with a coverslip. Several adult specimens were available and the eye on one side was dissected from the head to ensure that the hairs on the inner margin of eye were more clearly visible.

Terminology follows that of Sæther (1980) and Langton \& Pinder (2007) for the adult male, and Sæther (1980) and Langton (1991) for the pupal exuviae. Keys to separate adult males and pupal exuviae of the new genus from other similar genera are provided.

\section{RESULTS}

\section{Stygocladius gen. nov.}

Type-species: Stygocladius multisetosus sp. nov. by present designation.

Diagnostic characters: The adult male is separable from other Orthocladiinae genera by the following combination of characters: eyes bare, with dorsomedial extention; clypeus subequal in width to pedicel; wing normally developed, without pigmentation; wing membrane bare (with fine punctation); squama with setae; scutal hump absent; acrostichals present, never hooked or scalpellate, starting close to anterior margin of scutum; Epimeron II and posterior mesanepisternum without setae; lateral spines of spurs adpressed; hind tibial comb present; pulvilli absent; anal point broader at base, without setae or microtrichia; gonostylus simple; virga moderate to well developed; inferior volsella with both dorsal and ventral elongated triangular lobes. The pupa differs from all other genera by having pedes spurii B present on segments I and II and having an anal lobe with both a fringe and more than 3 anal macrosetae.

\section{Generic Diagnosis \\ Adult male}

Medium sized species, wing length $2.60-2.75 \mathrm{~mm}$.

Antenna. Antenna plumose, with 13 flagellomeres; groove beginning at segment 5 ; sensilla chaetica on flagellomeres 2 , 3 and 13 (distally); apex of antenna without subapical seta. AR greater than 1.9 .

Head. Eyes bare, with wedge-shaped dorsomedial extension. Temporal setae well developed, with both inner and outer verticals. Tentorium very broad in basal half, tapering medially to narrow finger-like apex. Clypeus subequal in width to pedicel, with setae. Palp 5 segmented, apex of segment 3 with 3-4 sensilla clavata.

Thorax. Antepronotum well developed, lobes meeting medially along short suture. Scutal hump absent. Antepronotal setae on posterior lobe only, acrostichals present (never hooked or scalpellate), weakly developed; dorsocentrals present; humerals absent; prealars present; supraalars absent; scutellars present. Preepisternum bare.

Wing. Membrane without setae, with fine punctation. Anal lobe distinctly protruding. Costa not or slightly extended (apparently without notch in wing membrane - see discussion). $\mathrm{R}_{4+5}$ ending distal to end of $\mathrm{M}_{3+4}$. FCu slightly distal to $\mathrm{RM}$. $\mathrm{Cu}_{1}$ curved. $\mathrm{R}$ with setae, remaining veins bare. Squama fully fringed.

Legs. Hind tibial comb present. Pseudospurs present. Sensilla chaetica apparently absent. Tarsomeres 4 and 5 cylindrical. Pulvilli absent.

Abdomen. Tergites and sternites with many irregularly scattered setae.

Hypopygium. Anal tergite varying from nearly subtriangular to semicircular; Anal point bare. Virga present, with 4-6 spines. Gonocoxite bearing a long extended inferior volsella, which is composed of two elongated triangular lobes, dorsal lobe larger than ventral lobe; dorsal lobe with nose-like apex. Gonostylus swollen distally, bearing an unusual double inner apical margin, crista dorsalis present, consisting of two crests situated basally and distally; megaseta of moderate length.

\section{Adult female}

As in male except:

Wing length $2.60 \mathrm{~mm}$.

Antenna. Antenna 6-segmented, bearing a darkened last flagellomere. AR greater than 0.7 .

Head. Palp 5-segmented, with intersegments 1-2 and 2-3 indistinct, intersegments 3-5 distinct, segment 4 triangular and bearing a truncate apical margin.

Thorax. Antepronotal setae present, on posterior lobe only, acrostichals present, weakly developed; dorsocentrals present; humerals absent; prealars present; supraalars absent; scutellars present. Preepisternum bare.

Wing. $R, R_{1}$ and $R_{4+5}$ with setae, remaining veins bare.

Legs. As in male.

Genitalia. Gonapophysis VIII with a wide, nearly rounded and non projecting ventrolateral lobe, covering most of the dorsomesal lobe; apodeme lobe indistinct. Tergite IX divided into two distinct protrusions (in contact medially). Two seminal capsules, similar in size to cerci, round, darkly sclerotised in apical two-thirds, with broad triangular neck. Spermathecal ducts relatively straight with slight bend. Cercus moderate in size.

\section{Pupa}

Medium sized, $4.5 \mathrm{~mm}$ long.

Cephalothorax. Frontal apotome rugulose, with rugulose warts, 
frontal setae present (not on cephalic tubercles). Thoracic horn present, simple and elongate, with spinulae (especially basally and on inner margin). Setation comprises 1 median and 2 lateral antepronotals, 3 precorneals and 4 dorsocentrals. Wing sheath without pearl row or nose.

Abdomen. Tergite I bare; II-VIII with shagreen, strongly developed posteriorly on II, extensive on III-VI, less extensive on VII, VIII-IX with weak spinules restricted anteriomedially. Conjunctive of V/VI with 2 rows of spinulae (spinulae smaller in female exuviae). Sternite I- VIII bare of shagreen. Tergite II without caudal hooklets, with a raised protuberance posteriorly with larger spinules. Pedes spurii A present. Pedes spurii B present on segments I and II. Distinct blackish apophyses present on apical border of segments II-VIII. Segment I with 1 L seta, II-VI each with 3 L setae, VII with 4 taeniate LS setae, VIII with 5 taeniate LS setae. Sexual dimorphism not evident on posterior margin of sternite VIII. Anal lobe with a well developed fringe (39 to 53 taeniate setae) and 5-10 macrosetae.

\section{Etymology}

The generic name Stygocladius is derived from Stygius, i.e. from the River Styx in the underworld of Greek mythology, referring to the habitat of the immatures which occur deep within rock fissures and-cladius, a typical ending for many genus-group names in the Orthocladiinae.

\section{Stygocladius multisetosus sp. nov.}

(Figures 1, 3-20, 22-28)

Heterotrissocladius sp. 1: Moubayed (1998), Moubayed-Breil (2007), Moubayed et al. (2007).

\section{Type material}

Holotype (mounted on 2 slides): ALGERIA: Boubhir Wadi, karstic helocrenes, altitude $220 \mathrm{~m}, 22$. iii.2001 (1 male pharate), A. Lounaci.

Paratypes: FRANCE: Hérault, Domain of Roque-Haute, basaltic helocrenes, altitude 35-30 m, 11-12.iv.1998, (1 male adult, 1 male pharate), J. Moubayed-Breil; same locality, 12.iii.1996 (2 male pupal exuviae, 3 female pupal exuviae), J. Moubayed-Breil; ALGERIA: same locality and date as holotype (6 male pharates, 1 female pharate), A. Lounaci; same locality, 22.iii.2001 (1 male pupal exuviae, 3 female pupal exuviae), A. Lounaci.

Holotype male and 3 Paratypes (1 male pharate, 1 female pharate, 1 male pupal exuviae) are deposited in the National Museum of Ireland, Kildare Street, Dublin 2, Ireland. Remaining paratypes are deposited in the collection of the senior author.

More details on the sampling sites in Algeria are given in Moubayed-Breil et al. (2007) and for France see Moubayed (1998) and Moubayed-Breil (2007).

\section{Etymology}

The species name multisetosus, means "many setae", referring to the presence of numerous macrosetae on the anal lobe of the

pupal exuviae.

\section{Description}

Adult male ( $\mathrm{n}=5$ ) (Figures 1, 3-10)

Medium sized species. Total length $4.30-4.90 \mathrm{~mm}$. Antenna length 1.14-1.17 mm. Wing length $2.60-2.75 \mathrm{~mm}$. General colouration contrasting brown to dark brown. Head dark brown. Mesonotal bands of thorax dark brown as are other parts of the thorax. Abdomen medium brown to dark brown. Legs brown, profemur dark brown, proximal and distal apices of femora and tibia blackish; tarsomere 5 of $\mathrm{P}_{1}-\mathrm{P}_{3}$ medium brown.

Head. Antenna 13-segmented with distinct division between segments; ultimate flagellomere 775-785 $\mu \mathrm{m}$ long; groove beginning at segment 5 ; sensilla chaetica on segments 2, 3 and 13 (distally); $\mathrm{AR}=1.96-2.21$. Eyes bare, with hairs on apical inner margin (Figure 1; Figure 2 - Heterotrissocladius marcidus (Walker), included for comparison). Temporal setae 11-13 including 7-8 inner and 4-5 outer verticals. Coronal triangle well open anteriorly, bearing 4 small setae confined in 2 rows. Clypeus subequal in width to pedicel, with 12-18 setae, maximal width $190-220 \mu \mathrm{m}$, minimal width $120-125 \mu \mathrm{m}$. Palp 5-segmented; lengths of segments (in $\mu \mathrm{m}$ ): 70-85, 170-195, 250$320,220-260,365-370$; third palpomere with 3-4 apical sensilla clavata.

Thorax. With 11-12 antepronotal setae (on posterior lobe only); acrostichals 16-18, short and weak nearly reaching antepronotum; dorsocentrals erect, 10-11; humerals 0; prealars 6; supraalars 0 ; scutellars 7-8 in a single row. Preepisternum bare.

Wing (Figure 4). Membrane bare, with fine punctation. Brachiolum with 2-3 setae and 5 sensilla campaniformia, Tegula with 5 sensilla campaniformia, Axillary sclerite I with 5 setae, $R$ with 9-11 setae, remaining veins bare. Anal lobe well developed, distinctly protruding. Squama with $25-28$ setae in 1-2 rows.

Legs. Spur of front tibia $53 \mu \mathrm{m}$ long, spurs of middle tibia43 and $35 \mu \mathrm{m}$ long, spurs of hind tibia $75 \& 26 \mu \mathrm{m}$ long; hind tibia comb with 12 setae. Sensilla chaetica not evident (apparently absent). Pseudospurs present on tarsomere 1 of mid and hind legs.

Length (in $\mu \mathrm{m}$ ) and proportions of legs:

\begin{tabular}{ccccccc}
\hline & $\mathrm{fe}$ & $\mathrm{ti}$ & $\mathrm{ta}_{1}$ & $\mathrm{ta}_{2}$ & $\mathrm{ta}_{3}$ & $\mathrm{ta}_{4}$ \\
\hline $\mathrm{P}_{1}$ & 1025 & 1075 & 830 & 490 & 135 & 115 \\
$\mathrm{P}_{2}$ & 995 & 1100 & 510 & 320 & 280 & 240 \\
$\mathrm{P}_{3}$ & 1030 & 1280 & 885 & 470 & 360 & 225 \\
\hline & $\mathrm{ta}_{5}$ & $\mathrm{LR}$ & $\mathrm{BV}$ & $\mathrm{SV}$ & $\mathrm{BR}$ \\
\hline $\mathrm{P}_{1}$ & 105 & 0.77 & 3.47 & 2.53 & 2.8 \\
$\mathrm{P}_{2}$ & 215 & 0.46 & 2.47 & 4.11 & 2.7 \\
$\mathrm{P}_{3}$ & 185 & 0.69 & 2.58 & 2.61 & 2.85 \\
\hline
\end{tabular}


Hypopygium. Dorsal and ventral view (Figure 5). Anal tergite varying from nearly subtriangular to semicircular, bearing 28-34 setae, 14-17 on each side of anal point. Anal point entirely bare, 58-65 $\mu \mathrm{m}$ long, 8-10 $\mu \mathrm{m}$ wide, width at base 31-33 $\mu \mathrm{m}$; slender and mostly of equal width, slightly tapering towards rounded apex; distance between apex of anal point and tip of dorsal lobe of inferior volsella includes intra-specific variations. Virga 41-46 $\mu \mathrm{m}$ long, consisting of 4-6 spines of equal size, basally fused. Gonocoxite $320-330 \mu \mathrm{m}$ long bearing an extended inferior volsella, which consists of two elongated triangular lobes (Figures 5-7), dorsal lobe is larger than ventral lobe, dorsal lobe 127-130 $\mu \mathrm{m}$ long, 50-55 $\mu \mathrm{m}$ wide, ventral lobe 130-135 $\mu \mathrm{m}$ long, 33-37 $\mu \mathrm{m}$ wide; dorsal lobe with nose-like apex; outer margin of dorsal lobe shows some intra-specific variation, which may be straight to the apex (Figure 6) or weakly swollen in the distal half (Figures 5 and 7). Gonostylus (Figures 5, 8-10) 120-125 $\mu \mathrm{m}$ long; crista dorsalis consists of two transparent crests bent inwards and outwards (Figures 810), proximal crest is smaller and bent outwards, distal crest is bigger and moderately bent inwards dorsally; basal margin of gonostylus swollen distally, bearing a transparent crest, which reaches the megaseta from behind (more visible (Figures 5,8) when the gonostylus is up against the gonocoxite). Variation in the shape of the gonostylus is dependant on its proximity to the gonocoxite, up against the gonocoxite (Figures 5, 8), intermediate (Figure 9) or displaced downward (Figure 10). Megaseta 23-25 $\mu \mathrm{m}$ long and nearly transparent.

Adult female $(\mathrm{n}=1)$ (Figures 11-20)

Colouration as in the male, except for the antenna, which is whitish to medium brown. Antenna with segments 1-2 whitish, segments 3-5 light brown to medium brown, last segment medium brown to brown; segments 2 brownish apically, segments 3-5 brownish basally and apically. Total length 4.80 $\mathrm{mm}$. Wing length $2.60 \mathrm{~mm}$. Antenna length $0.36 \mathrm{~mm}$.

Head. Antenna (Figures 11-12) 6-segmented; length of segments (in $\mu \mathrm{m}$ ): 45, 37, 40, 42, 44, 150; ultimate flagellomere (Figure 12) with about 9-11 sensilla chaetica. AR 0.72. Eyes bare, hairs present on apical inner margin. Temporal setae 8 including 6 inner and 2 outer verticals. Clypeus with about 18 setae. Palp (Figures 13-14) 5-segmented, lengths of segments 2 to 5 (in $\mu \mathrm{m}$ ): $38,43,78,131$; intersegments $1-2$ and 2-3 indistinct; intersegments 3-5 distinct; segment 4 triangular and bearing a truncate apical margin; sensilla clavata absent on segment 3 ; presence of sensilla chaetica on last segment.

Thorax. In dorsal view (Figure 15). With 11 antepronotal setae (on posterior lobe only); acrostichals 16, about $18 \mu \mathrm{m}$ long, short and weak nearly reaching antepronotum; dorsocentrals 10; humerals 0; prealars 6; supraalars 0; scutellars 8 in a single row. Preepisternum bare.

Wing. Wing length $2.60 \mathrm{~mm}$. $R$ with 11 setae, $R_{1}$ with $7-8$ setae, $\mathrm{R}_{4+5}$ with 10-11 setae, remaining veins bare. Squama with 28-29 setae.
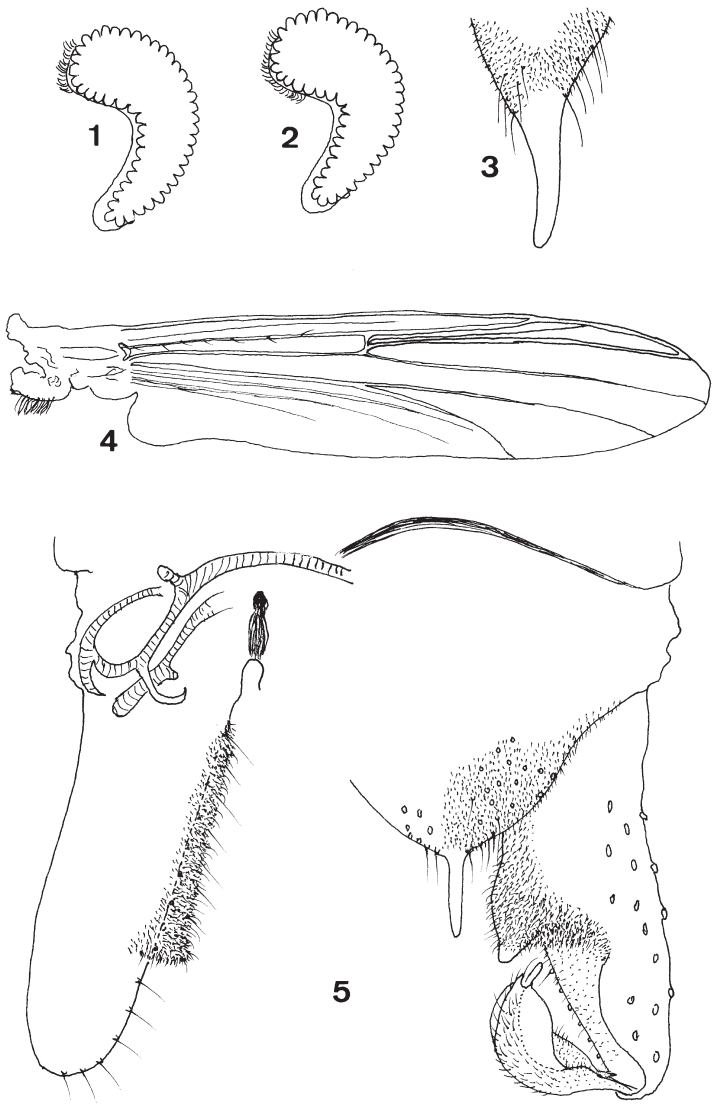

Figures 1-5. Stygocladius multisetosus gen. nov., sp. nov., male imago, 1, 3-5, Heterotrissocladius marcidus (Walker), 2. 1-2, Hairs on dorsal and lateral inner margin of eye: (1) S. multisetosus; (2) $H$. marcidus; (4) Wing of S. multisetosus;. (3 and 5) Hypopygium : (3) anal point in lateral view; (5) hypopygium in dorsal and ventral view.
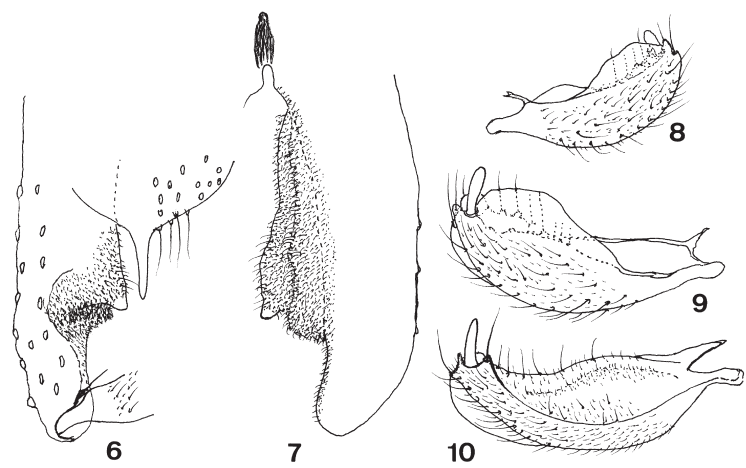

Figures 6-10. Stygocladius multisetosus gen. nov., sp. nov., male hypopygium: (6) dorsal view, (7) ventral view. Three aspects of gonostylus: (8) left, (9-10) right, showing proximal and distal crest of crista dorsalis and shape of distal margin with swollen hump. 


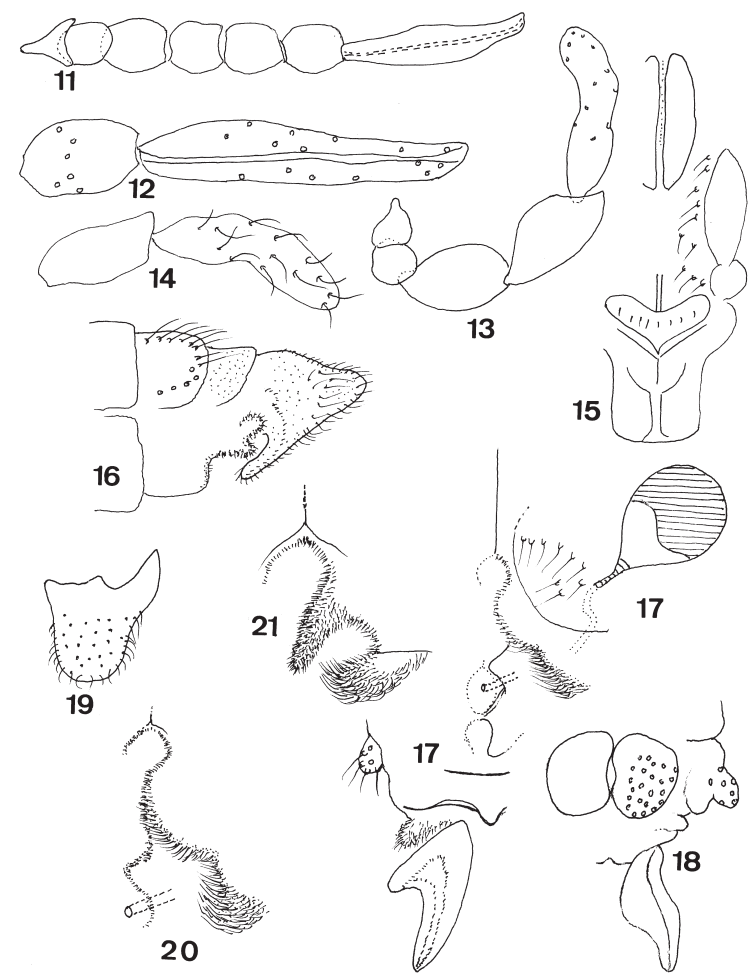

Figures II-2l. female imago, 11-20, Stygocladius multisetosus subgen. nov., sp. nov., 21, H. marcidus; (11) Antenna, (12) apical two antennal segments. (13) Palp, (14) apical two segments. (15) Thorax, dorsal view. Anal segment: (16) lateral view, (17) ventral view showing sternite IX, seminal sac and gonapophysis VIII; (18) tergite IX in dorsal view, (19) Cercus. (20-21) Gonapophysis VIII: (20) S. multisetosus, (21) H. marcidus.

Legs. Spur of front tibia $58 \mu \mathrm{m}$ long, spurs of middle tibia 35 $\mu \mathrm{m}$ and $28 \mu \mathrm{m}$ long, spurs of hind tibia $71 \mu \mathrm{m}$ and $28 \mu \mathrm{m}$ long; hind tibia comb with 11 setae. Sensilla chaetica not evident (apparently absent). Pseudospurs present on tarsomere 1 of mid and hind legs.

Length (in $\mu \mathrm{m}$ ) and proportions of legs:

\begin{tabular}{ccccccc}
\hline & $\mathrm{fe}$ & $\mathrm{ti}$ & $\mathrm{ta}_{1}$ & $\mathrm{ta}_{2}$ & $\mathrm{ta}_{3}$ & $\mathrm{ta}_{4}$ \\
\hline $\mathrm{P}_{1}$ & 653 & 755 & 372 & 210 & 167 & 128 \\
$\mathrm{P}_{2}$ & 705 & 862 & 413 & 274 & 186 & 145 \\
$\mathrm{P}_{3}$ & 749 & 847 & 542 & 298 & 231 & 178 \\
\hline & $\mathrm{ta}_{5}$ & $\mathrm{LR}$ & $\mathrm{BV}$ & $\mathrm{SV}$ & $\mathrm{BR}$ \\
\hline $\mathrm{P}_{1}$ & 131 & 0.49 & 3.80 & 3.78 & 1.75 \\
$\mathrm{P}_{2}$ & 132 & 0.48 & 2.69 & 3.79 & 1.20 \\
$\mathrm{P}_{3}$ & 137 & 0.64 & 2.53 & 2.94 & 2.43 \\
\hline
\end{tabular}

Genitalia. In lateral (Figure 16), ventral (Figure 17) and dorsal view (Figure 18). Notum $129 \mu \mathrm{m}$ long, ramus $59 \mu \mathrm{m}$ long;

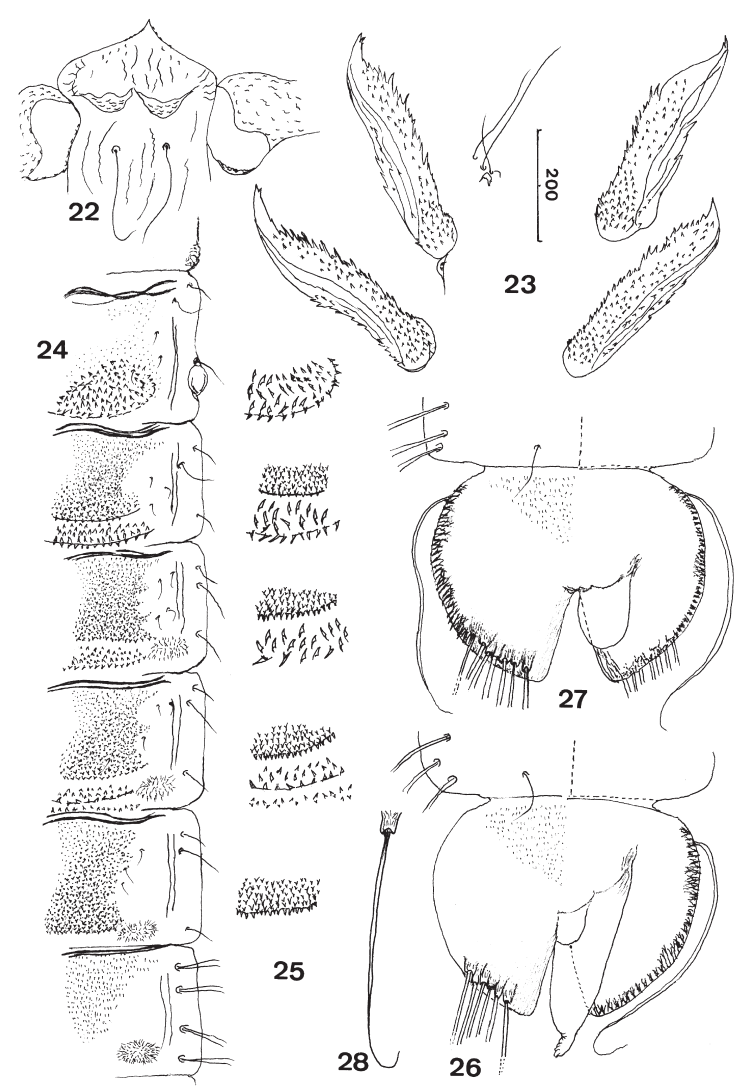

Figures 22-28. Stygocladius multisetosus gen. nov., sp. nov., pupal exuviae: (22) Frontal apotome; (23) 4 aspects of thoracic horn, left and right; (24) armament pattern of abdominal segments I-VII; (25) details of armaments on tergites II-VI; (26) anal segment of male in dorsal and ventral view; (27) anal segment of female; (28) anal macrosetae. Scale in $\mu \mathrm{m}$.

notum ratio 2.18. Gonapophysis VIII (Figures 17, 20) bearing a very wide rounded ventrolateral lobe which covers most of the dorsomesal lobe and the indistinct apodeme lobe; ventrolateral lobe is not projecting as in Heterotrissocladius species (e.g. H. marcidus, Figure 21). Seminal capsules (one on each side) pearl-shaped and almost half sclerotized, $135 \mu \mathrm{m}$ long, 108 $\mu \mathrm{m}$ wide. Sternite VIII with 9-10 setae. Gonocoxite globular, with 7-8 setae. Genitalia in dorsal view (Figure 18); tergite IX divided into two distinct protrusions, in contact medially, each subrectangular to suboval in shape, bearing 19-21 setae. Cercus, 200-220 $\mu \mathrm{m}$ long, in side view (Figure 19).

Pupal exuviae $(\mathrm{n}=11)$ (Figures 22-28)

Total length: 4.45-4.95 mm (male), 4.80-5.50 mm (female); abdomen length $3.40-3.90 \mathrm{~mm}$ (male), $3.80-4.50 \mathrm{~mm}$ (female). Colour brown in general except for the frontal apotome and genital sac, which are transparent; specimens from southern France are somewhat darker than those from Algeria; abdomen and anal segment brownish.

Cephalothorax. Frontal apotome including frontal warts, antennal sheaths and thoracic suture are covered with granulations. 
Frontal apotome rugulose (Figure 22) with distinctly domed and broadened anteriomedian rugulose warts; frontal setae (not on tubercules) bristle-like, 125-135 $\mu \mathrm{m}$ long. Thorax with 2 lateral antepronotals 195 and $225 \mu \mathrm{m}$ long, and 1 median antepronotal 83-85 $\mu \mathrm{m}$ long. Thoracic horn (Figure 23) 435$495 \mu \mathrm{m}$ long, base covered with dense spinulae, becoming less intense medially and apically, inner margin more toothed than the exterior margin; precorneal setae 205, 178 and $79 \mu \mathrm{m}$ long. Dorsocentrals consist of 4 stout setae 68-75 $\mu \mathrm{m}$ long, confined in a single row parallel to the thoracic suture; distance between dorsocentrals progressively increasing from Dc1-Dc2 to Dc3Dc4 $(57$ to $97 \mu \mathrm{m})$; distance between dorsocentral row and thoracic suture 153-171 $\mu \mathrm{m}$. Wing sheaths without pearl row.

Abdomen. Shagreen absent on tergite I. Pedes spurii B located posterolaterally on segments I-II: flattened and composed of granules on segment I, 75-85 $\mu \mathrm{m}$ high, 5-10 $\mu \mathrm{m}$ wide; pale to nearly transparent and well developed on segment II, much higher than wide (male, $110 \mu \mathrm{m}$ high, $75 \mu \mathrm{m}$ wide; female, 140 $\mu \mathrm{m}$ high, $125 \mu \mathrm{m}$ wide). Pedes spurii A present on segments IV-VII, suboval to subrectangular and exclusively composed of small concentric spinulae. Distinct blackish apophyses present on apical border of tergites and sternites II-VIII. Armament pattern of tergites I-VII (Figures 24-25): shagreen present anteriorly and medially on tergites II, VII and VIII; tergite II with only one posteromedian group of short projecting spines. Tergites III-VI with anteromedian group of points, gradually becoming larger posteriorly on each segment; tergites III-V with a posterior transverse band of short spines often projecting upwards. Sternite I- VIII bare of shagreen. Intersegment V/VI with a transverse band of spinules occasionally projecting upwards. Lateral setae on segments I-VI: I with 1 , II-V with 3, VI with 3 (occasionally 4). Lateral filaments (taeniae) on segments VII-VIII: VII with 4, VIII with 5 (occasionally 6). Posterolateral corner of segment VIII rounded. Posterior margin of sternite VIII similar in both male and female, no indication of sexual dimorphism. Anal segment (Figures 26-27, male and female), 480-490 $\mu \mathrm{m}$ long (male), 505-515 $\mu \mathrm{m}$ long (female), shagreen present anteriorly in both male and female. Genital sac 305-315 $\mu \mathrm{m}$ long (male), 245-255 $\mu \mathrm{m}$ long (female); genital sac of male overreaching tip of anal segment by $45-55 \mu \mathrm{m}$. Fringe with taeniate setae, 39-43 (in male), $44-53$ (in female). Anal lobe with faint granulations dorsally. Macrosetae (Figure 28) 470-520 $\mu \mathrm{m}$ long, stout and inserted on well developed tubercles, curved and pointed apically; with an average of 5 to 7 (occasionally 9 to 10) on each anal lobe.

Larva: Unknown.

\section{DISCUSSION}

In the key to Holarctic male Orthocladiinae (Cranston et al. 1989) Stygocladius keys unsatisfactorily to couplet 79, which includes "Antillocladius (part. arcuatus Sæther)" and
"Orthocladius (part.)". In Stygocladius the combination of diagnostic features in the adults and pupae indicates placement in the Heterotrissocladius group of genera as defined in Sæther (2000). This group includes Aagardia Sæther, 2000, Heterotrissocladius Spärck, 1923, Parametriocnemus Goetghebuer, 1932, Paraphaenocladius Thienemann, 1924, Paratrissocladius Zavřel, 1937, Platysmittia Sæther, 1982 and Psilometriocnemus Sæther, 1969. Stygocladius shares two adult characters with Platysmittia, wing membrane bare (with fine punctation) and preudospurs present, which are not found in any of the other genera. The distinctly protruding anal lobe on the wing is otherwise found only in Heterotrissocladius and Paratrissocladius. In the wing of Stygocladius at the end of the costa there is a small gap, sometimes with a slight concavity, where a few fringe setae are missing - whether or not this constitutes a notch (sensu Sæther 1975) as found in Heterotrissocladius is unclear.

In Sæther et al. (2000) the pupal exuviae of Stygocladius keys to couplet 88 with two unsatisfactory options: either Psectrocladius (Psectrocladius) (part, i.e. P. (P.) barbimanus) or Propsilocerus. Within the Orthocladiinae the presence of more than three macrosetae as well as a fringe of setae on the anal lobe of the pupal exuviae is rare and is otherwise reported only in Psectrocladius (P.) barbimanus, Hydrobaenus olfa and in the genus Propsilocerus (Coffman et al. 1986, Zerguine and Rossaro 2010). Having more than three macrosetae has evolved independently in all these taxa and its occurrence is not indicative of a relationship between any of them. Preliminary molecular data in Cranston et al. (2012) indicates that Propsilocerus should be placed in the Prodiamesinae but no formal change removing it from the Orthocladiinae has yet been made pending further molecular analysis. Among Holarctic genera pedes spurii B on tergite I is otherwise only reported in some Halocladius s. str. Hirvenoja, 1973. Utilizing the same pupal characters in the relationships between the Heterotrissocladius group of genera (Sæther 2000) shows that Stygocladius is closest to Paratrissocladius based on the following shared features: sexual dimorphism absent on sternite VIII, hooklets on tergite II absent, pedes spurii A present. The diagnoses and data alluded to above for both the adults and pupae indicate that Stygocladius is most probably the sister group to Paratrissocladius with these two together forming the sister group to Heterotrissocladius.

\section{Keys to distinguish Stygocladius from other genera}

Adult males - keys in Cranston et al. (1989) to couplet 79 with two unsatisfactory options, either Antillocladius (part. arcuatus Sæther) or Orthocladius (part.). Couplet 79 is expanded below to include Stygocladius.

79. Costa not or very slightly extended (Figure 4). Anal point lacking setae (Figure 5)

Stygocladius Moubayed-Breil, Ashe \& Langton 1 sp.: S. multisetosus Moubayed-Breil, Ashe \& Langton, 
Palaearctic (Algeria, France).

- Costa strongly extended. Anal point with setae $79 \mathrm{a}$

79a. Costa strongly extended, reaching close to apex of wing. Anal point strongly developed, broadly based, evenly tapering, setose to apex. (Figure 9.4)

Antillocladius (part. arcuatus Sæther)

- Costa less strongly extended, not approaching apex of wing. Anal point not as strong or broadly based as above Orthocladius (part.)

Pupal exuviae - only includes exuviae with both an anal fringe and more than three anal macrosetae starting with couplet 58 in Sæther et al. (2000).

58. Anal lobes with 3 macrosetae; macrosetae are usually all long and hair-like, but may be short, spine-like or unequal in length 59

[not keyed - see Sæther et al. (2000)]

- Anal lobes with 0-1 or 5-10 macrosetae 87

87. Anal lobes with 0-1 macrosetae .. 89

[not keyed - see Sæther et al. (2000)]

- Anal lobes with 5-10 macrosetae $88 \mathrm{a}$

88a Tergites IV-VI with 1 or 2 (usually 2) median spine patches

Psectrocladius (Psectrocladius) barbimanus (Edwards)

Tergites IV-VI without median spine patches .. $.88 \mathrm{~b}$

88b Tergite II without posterior hooklets; Pedes spurii B present on Tergites I and II Stygocladius multisetosus MoubayedBreil, Ashe \& Langton Tergite II with posterior hooklets; Pedes spurii B present only on Tergite II . $.88 \mathrm{c}$

88c Frontal setae weak on praefrons or absent; Pedes spurii A well developed on sternite IV, absent or weak on V, VI or VII; Thoracic horn apically bifid or with irregular division, usually bent medially

Propsilocerus Kieffer

Frontal setae present on frontal apotome; Pedes spurii A present on sternites IV-VII; Thoracic horn not bifid apically, with pointed apex, not bent medially

Hydrobaenus olfa Zerguine \& Rossaro

\section{Ecology and geographical distribution}

Adults, pharates and pupal exuviae of Stygocladius multisetosus are currently only known from basaltic helocrenes in southern France and karstic helocrenes in Algeria. The Domain of RoqueHaute locality in southern France is a small, shallow spring-fed, temporary lake and Boubhir Wadi in Algeria is a river valley with active springs for a few months - both habitats on the surface are dry for most of the year. In these helocrenes the immature stages are stygobiontic and their survival and growth is strongly influenced by a combination of: (1) alternation between very high (flooding) and zero availability (drought) of surface water; (2) low variation in temperature; (3) permanence of wet conditions in deeper sub-surface layers; and (4) annual rhythm between a short wet season and a long dry season. The new species is likely to be more widespread at least in the western Mediterranean and can be expected from helocrene habitats elsewhere in the region which also includes eastern Spain, western Italy, Tunisia, Morocco and some of the larger islands (i.e. Corsica, Sardinia, Sicily). The genus Stygocladius can be expected to occur more widely in the Palaearctic and its eventual discovery in the Nearctic would not be surprising.

Associated hygropetric or madicolous species encountered in the same localities include: Bryophaenocladius aestivus (Brundin, 1947), B. nidorum (Edwards, 1929), B. vernalis (Goetghebuer, 1921), Chaetocladius (Chaetocladius) melaleucus (Meigen, 1818), C. (C.) perennis (Meigen, 1830), Heterotrissocladius marcidus (Walker, 1856), Parametriocnemus stylatus (Spärck, 1923) and P. valescurensis Moubayed \& Langton, 1999.

\section{ACKNOWLEDGMENTS}

The authors are grateful to Dr A. Lounaci and Dr D. LounaciDaoudi (University of M. Mammeri, Tizi-Ouzou, Algeria) for their kindness in providing material of the new species from Boubhir Wadi in northern Algeria.

\section{REFERENCES}

Coffman WP, Cranston PS, Oliver DR, Sæther OA. 1986. The pupae of Orthocladiinae (Diptera: Chironomidae) of the Holarctic region-Keys and diagnoses. In: Wiederholm, T. (ed.). Chironomidae of the Holarctic region. Keys and diagnoses. Part 2 - Pupae. Entomologica Scandinavica Supplement 28: 147-296.

Cranston PS, Hardy NB, Morse GE. 2012. A dated molecular phylogeny for the Chironomidae (Diptera). Systematic Entomology 37: $172-188$.

Cranston PS, Oliver DR, Sæther OA. 1989. The adult males of Orthocladiinae (Diptera: Chironomidae) of the Holarctic region - Keys and diagnoses. In: Wiederholm, T. (ed.). Chironomidae of the Holarctic region. Keys and diagnoses. Part 3 - Adult males. Entomologica Scandinavica Supplement 34: 164-352.

Langton PH. 1991. A key to pupal exuviae of the West Palaearctic Chironomidae. Privately published. Huntingdon, England, 386 pp.

Langton PH, Pinder LCV. 2007. Keys to the adult male Chironomidae of Britain and Ireland. Volume 1 (Pp: 1-239) and volume 2 (Pp: 1-168). Freshwater Biological Association, Scientific Publication, $\mathrm{N}^{\circ} 64$.

Moubayed J. 1998. The basaltic table of Roque-Haute (Herault, France): main characteristics and global quality of the wetland area. Ecologia Mediterranea 24(2): 185-206. 
Moubayed-Breil J. 2007. Non-biting midges from Continental France: new records, faunal and biogeographical outline (Diptera, Chironomidae). Ephemera 9(1): 17-32.

Moubayed-Breil J, Lounaci A, Lounaci-Daoudi D. 2007. Non-biting midges from Algeria, North Africa (Diptera, Chironomidae). Ephemera 8(2): 93-99.

Sæther OA. 1975. Nearctic and Palaearctic Heterotrissocladius (Diptera: Chironomidae). Bulletin of the Fisheries Research Board of Canada 193: 1-67.

Sæther OA. 1980. Glossary of chironomid morphology terminology (Diptera: Chironomidae). Entomologica Scandinavica Supplement 14: 1-51.

Sæther OA. 2000. Aagaardia, a new Holarctic orthoclad genus (Diptera: Chironomidae). Aquatic Insects 22: 177-196.

Sæther OA, Ashe P, Murray DA. 2000. Family Chironomidae. Pp 113-334. In: Papp, L. and Darvas, B. (eds.) Contributions to a Manual of Palaearctic Diptera (with special reference to the flies of economic importance). Volume 4 (Appendix) A.6. Science Herald, Budapest.

Zerguine K, Rossaro B. 2010. A new species of Hydrobaenus Fries, 1830 (Diptera, Chironomidae) from Algeria. Zootaxa 2507: 37-43. 